Respiration 2016;92:356-358

DOI: $10.1159 / 000450758$

\section{Bilateral Endoscopic Lung Volume Reduction in Patients with Severe Emphysema}

\begin{abstract}
Franziska C. Trudzinski ${ }^{a}$ Philipp M. Lepper ${ }^{\mathrm{a}}$ Daniela Leppert ${ }^{\mathrm{b}}$ Frank Langer ${ }^{c}$ Christian Lensch $^{a}$ Monika Flaig ${ }^{a}$ Robert Bals ${ }^{a}$ Heinrike Wilkens ${ }^{a}$ Peter Minko $^{d}$ Sebastian Fähndrich ${ }^{a}$

a Department of Internal Medicine V - Pneumology, Allergology, and Critical Care Medicine, and Departments of ${ }^{\mathrm{b}}$ Thoracic and Cardiovascular Surgery, ' Nuclear Medicine, and ${ }^{\mathrm{d}}$ Diagnostic and Interventional Radiology, University Hospital of Saarland, Homburg, Germany
\end{abstract}

Key Words

Chronic obstructive pulmonary disease $\cdot$ Endobronchial valves · Severe emphysema

\section{Abstract}

Reducing hyperinflated areas in chronic obstructive pulmonary disease, either surgically or endoscopically, leads to improvement of functional parameters. It is unclear if bilateral treatment with endobronchial valves (EBV) aiming at total lobar occlusion is beneficial. The aim of this study was to assess the results after staged bilateral endoscopic treatment with EBV. This is a retrospective analysis of patients with severe airflow obstruction, who were treated bilaterally with EBV in two stages, aiming at subsequent atelectasis. Pre- and postintervention lung function parameters, the 6-minute walk test (6-MWT), complications, and followup were recorded. Sixteen patients were treated bilaterally in two stages. There was an overall improvement in lung function from baseline to second-treatment follow-up with an increase in $\mathrm{FEV}_{1}$ (23.57-29.21\% of predicted) and a decrease in residual volume (299.21-240.10\% of predicted) and total lung capacity (140.78$128.71 \%$ of predicted). The 6 -MWT improved up to $54 \mathrm{~m}$. After each procedure, 9 of 16 patients (56.25\%) developed an atelectasis of the target lobe. Overall, pneumothorax occurred in 8 of 32 procedures (25\%). No patient died. Patients benefitted from the first EBV treatment. The second treatment did not lead to marked improvements compared to the first treatment. Bilateral lung volume reduction with valves is possible; however, the current results seem not to justify bilateral valve treatment as a routine approach.

(C) 2016 S. Karger AG, Basel

Advanced chronic obstructive pulmonary disease (COPD) is a disabling disease. Pulmonary rehabilitation is known to provide clinically and statistically significant improvements in quality of life and functional exercise capacity, which are outcomes that few other treatments can provide. Hence, symptomatic therapy options are limited for these patients. Reducing hyperinflated areas either surgically or endoscopically has been shown to improve lung function and exercise capacity $[1,2]$. However, in the National Emphysema Treatment Trial (NETT), an early mortality rate of about $5 \%$ was reported [1]. This has led to a quest for minimally invasive methods. The value of endoscopic lung volume reduction (eLVR) is unclear, and none of the methods has found a clear place in patient treatment as yet. The recently published sham-controlled trial on eLVR using endobronchial valves (EBV) has raised additional questions [3], especially as 2 patients of the $25(8 \%)$ treated with valves died. While the interventional group in this study experienced some benefits regarding lung function and exercise capacity, one question from an accompanying editorial was whether patients had a double benefit (and possibly double morbidity and mortality) if they were treated bilaterally [4].

In fact, we hypothesized that patients can benefit from bilateral valve implantation provided that they have a bilateral treatment target (heterogeneous perfusion and no collateral ventilation). The rationale behind this approach was that parenchyma destruction and hyperinflation are almost always bilateral; thus, we performed bilateral eLVR in analogy to the surgical bilateral approach [5]. We treated 16 patients (mean age $60.10 \pm 8.26$ years, $50 \%$ male) bilaterally with EBV in two stages according to an institutional protocol. Staged treatment is mandatory due to the risk of pneumothorax.

One prerequisite for treatment was established maximal pharmacological treatment. DLCO $<20 \%$ of predicted (pred.) and homogeneous distribution of emphysema were no exclusion criteria to treatment unless perfusion scintigraphy also showed a homogeneous distribution of perfusion, rendering the selection of a target lobe impossible.

Prior to the procedure, all patients underwent functional testing including postbronchodilator lung function, 6-minute walk test (6-MWT), single-breath DLCO, and blood gas analysis. During the 6-MWT, supplemental oxygen was administered through a nasal cannula, if necessary, to maintain the arterial oxygen saturation at $90 \%$ or better. These standards were kept before and after eLVR.

All patients had previously smoked cigarettes, but our protocol requires patients to quit smoking more than 3 months before the intervention. One prerequisite for treatment was established maximal pharmacological treatment (at least a long-acting $\beta$-agonist, long-acting muscarinic antagonist, or inhaled corticosteroid). Pulmonary rehabilitation was not a prerequisite for treatment.

Ventilation and perfusion scintigraphy (V/Q scan) or perfusion scan only and computed tomography plus assessment of collateral ventilation using a catheter system (Chartis; Pulmonx, Redwood City, Calif., USA) to determine the target lobe were mandatory. Successful eLVR was defined as atelectasis visible in chest X-ray or computed tomography. Generally, interventions were done in total intravenous anesthesia, and patients were intubated and mechanically ventilated. The EBV used in these patients was a one-way sili-

\section{KARGER}

E-Mail karger@karger.com

www.karger.com/res
2016 S. Karger AG, Basel

$0025-7931 / 16 / 0925-0356 \$ 39.50 / 0$
Philipp M. Lepper, MD

Department of Internal Medicine V - Pneumology, Allergology, and

Critical Care Medicine, University Hospital of Saarland

Kirrberger Strasse 1, DE-66421 Homburg (Germany)

E-Mail philipp.lepper@uks.eu 
Table 1. Functional characteristics and complications of patients prior to and after first and second eLVR

\begin{tabular}{|c|c|c|c|c|c|c|c|c|}
\hline & \multicolumn{4}{|l|}{ First eLVR } & \multicolumn{4}{|l|}{ Second eLVR } \\
\hline & baseline & follow-up & $\Delta$ & $\mathrm{p}$ & baseline & follow-up & $\Delta$ & $\mathrm{p}$ \\
\hline $\mathrm{FEV}_{1}$, liters & $0.62 \pm 0.31$ & $0.76 \pm 0.28$ & $+0.14^{\mathrm{b}}$ & 0.044 & $0.68 \pm 0.31$ & $0.74 \pm 0.28$ & +0.06 & 0.03 \\
\hline $\mathrm{FEV}_{1}, \%$ & $23.57 \pm 11.21$ & $29.37 \pm 12.01$ & +5.8 & 0.017 & $25.64 \pm 9.53$ & $29.21 \pm 10.38$ & +3.57 & 0.001 \\
\hline VC, liters & $1.85 \pm 0.92$ & $2.15 \pm 0.67$ & +0.30 & 0.031 & $2.21 \pm 1.09$ & $2.17 \pm 0.85$ & -0.04 & n.s. \\
\hline VC, \% & $53.09 \pm 19.27$ & $62.18 \pm 17.75$ & +9.09 & 0.031 & $60.41 \pm 19.8$ & $63.08 \pm 14.8$ & +2.61 & n.s. \\
\hline $\mathrm{RV}$, liters & $6.17 \pm 1.82$ & $4.58 \pm 1.15$ & $-1.59^{b}$ & 0.009 & $5.81 \pm 1.62$ & $5.14 \pm 1.57$ & -0.67 & n.s. \\
\hline $\mathrm{RV}, \%$ & $299.21 \pm 91.38$ & $224.11 \pm 38.51$ & $-75.1^{\mathrm{b}}$ & 0.005 & $278.12 \pm 78.44$ & $240.10 \pm 41.07$ & -38.02 & 0.04 \\
\hline TLC, liters & $8.08 \pm 2.11$ & $7.16 \pm 2.44$ & -0.92 & 0.044 & $7.91 \pm 1.91$ & $7.31 \pm 2.03$ & -0.6 & n.s. \\
\hline TLC, $\%$ & $140.78 \pm 25.0$ & $123.19 \pm 19.50$ & -17.59 & 0.030 & $138.31 \pm 128.71$ & $128.71 \pm 15.22$ & -9.6 & n.s. \\
\hline $\mathrm{RV} / \mathrm{TLC}$ ratio & $76.63 \pm 8.25$ & $68.80 \pm 8.26$ & -7.83 & 0.011 & $73.68 \pm 9.8$ & $71.30 \pm 8.08$ & -2.38 & n.s. \\
\hline 6-MWT, m $\left(\mathrm{n}=15^{\mathrm{a}}\right)$ & $247.69 \pm 72.27$ & $283.05 \pm 89.26$ & $+35.36^{\mathrm{b}}$ & n.s. & $302.27 \pm 99.37$ & $277.92 \pm 83.67$ & -24.35 & n.s. \\
\hline Atelelectasis, n/total n (\%) & - & $9 / 16(56.25)$ & & & - & $9 / 16(56.25)$ & & \\
\hline Pneumothorax, n/total n (\%) & - & $3 / 16(18.75)$ & & & - & $5 / 16(31.25)$ & & \\
\hline Time on chest tube, days & - & $10.22 \pm 3.3$ & & & - & $12.20 \pm 7.93$ & & \\
\hline
\end{tabular}

$\Delta=$ Difference between pre- and posttreatment is given in liters or meters, as appropriate; n.s. = statistically not significant ( $\mathrm{p} \geq$ 0.05). ${ }^{a}$ One patient refused the 6-MWT. ${ }^{\mathrm{b}}$ Minimal clinically important difference was reached $\left(\mathrm{FEV}_{1}>100 \mathrm{ml}\right.$; RV $>430 \mathrm{ml} ; 6-\mathrm{MWT}$ increase $>26 \mathrm{~m})$.

cone duckbill valve (Zephyr EBV; Pulmonx). All patients received a chest X-ray $1 \mathrm{~h}$ after the procedure (or earlier if symptoms occurred) to exclude a pneumothorax. The valves were placed unilaterally in lobar, segmental, or subsegmental bronchi based on the individual anatomic conditions with the intention to completely isolate the target lobe. All patients gave informed consent separately for both interventions. The analysis was approved by the institutional review board (Ärztekammer des Saarlandes; No. 35/15). The necessity for informed consent was waived by the institutional review board due to the retrospective nature of the study.

The mean $\mathrm{FEV}_{1}$ prior to the first intervention was $0.62 \pm 0.31$ liters, equaling $23.57 \pm 11.21 \%$ of pred. normal values, at a total lung capacity (TLC) of $8.08 \pm 2.11$ liters and $140.78 \pm 25.0 \%$ of pred. The residual volume $(\mathrm{RV})$ was $6.17 \pm 1.82$ liters, equaling $299.21 \pm 91.38 \%$ of pred.; thus, the RV/TLC ratio was increased to $76.63 \pm 8.25 \%$ of pred. Diffusing capacity DLCO for was $<20 \%$ in 10 patients and $31.6 \pm 9.03 \%$ in 6 patients. All patients presented with heterogeneous perfusion in the V/Q scan. Collateral ventilation could be excluded by Chartis measurement in 26 of 32 measurements $(81 \%)$.

There was an overall improvement in lung function after the first procedure with an increase in $\mathrm{FEV}_{1}(0.76 \pm 0.28$ liters, equaling $29.37 \pm 12.01 \%$ of pred.) and a decrease in RV ( $4.58 \pm 1.15$ liters, equaling $224.11 \pm 38.51 \%$ of pred.) and TLC (7.16 \pm 2.44 , equaling $123.19 \pm 19.50 \%$ of pred.). Subsequently, there was a reduction of the RV/TLC ratio $(68.80 \pm 8.26)$. These changes were statistically significant and reached minimal clinically important difference. The 6-MWT increased from $242.20 \pm 71.28 \mathrm{~m}$ to a peak of 302.27 $\pm 99.37 \mathrm{~m}$. Nine $(56.25 \%)$ patients had an atelectasis and $3(18.75 \%)$ patients had a pneumothorax after the procedure and required a chest tube.

The second treatment was performed $128.19 \pm 109.91(43-483)$ days after the procedure. Baseline data for the second intervention were as follows: $\mathrm{FEV}_{1} 0.68 \pm 0.31$ liters, equaling $25.64 \pm 9.53 \%$ of pred., RV $5.81 \pm 1.62$ liters, equaling $278.12 \pm 78.44 \%$ of pred., TLC $7.91 \pm 1.91$ liters, equaling $138.31 \pm 128.71 \%$ of pred., and RV/TLC $73.68 \pm 9.8$. After the second treatment, again, 9 patients had an atelectasis and $5(31.25 \%)$ patients developed a pneumothorax. Overall, 7 patients had a pneumothorax and 1 of those had a pneumothorax after each treatment. This is an overall pneumothorax rate of $25 \%$ ( 8 events in 32 procedures). All pneumothoraxes were treated with chest tube only and did not require surgical interventions.

Now, there was an increase in $\mathrm{FEV}_{1}$ from $0.68 \pm 0.31$ to $0.74 \pm$ 0.28 liters ( $25.64 \pm 9.53 \%$ to $29.21 \pm 10.38 \%$ of pred.) and a reduction of TLC and RV from $7.91 \pm 1.91$ to $7.31 \pm 2.03$ liters (138.31 $\pm 128.71 \%$ to $128.71 \pm 15.22 \%$ of pred.) and $5.81 \pm 1.62$ to $5.14 \pm$ 1.57 liters $(278.12 \pm 78.44 \%$ to $240.10 \pm 41.07 \%$ of pred.), respectively (table 1).

This retrospective analysis of 16 patients with advanced COPD showed that eLVR can clinically relevantly improve lung function and exercise capacity - after the first intervention. Even though the second intervention showed some lung function improvements, this did not translate into a functional benefit. Despite the fact that the second treatment was performed only $128.19 \pm 109.91$ (43483) days after the first intervention, patients were almost back to baseline. The second treatment at best consolidated the results of the first intervention. Additionally, we noted a considerable rate of pneumothoraxes.

One reason for this disappointing result might be the short interval between the intervention and the follow-up, which was only 4-6 weeks. This might not have been enough time for patients to recover from their procedure and admission, particularly those with a prolonged stay following pneumothorax. Another limitation is the fact that rehabilitation was not a prerequisite for treatment and that 3 patients had to be considered collateral ventilation positive. 
Another point might be that the disease progresses over time and that the lung areas that are not partially or fully atelectatic most likely tend to become emphysematic very quickly. An important finding might be that patients with advanced COPD might still improve despite functional bilobectomy in case they develop bilateral atelectasis. While our results are in line with previously published results [6], or even slightly better $[2,3,7]$, it has to be assumed that surgical lung volume reduction has the potential for greater functional improvements [8]. If ineligible for surgery, bilateral EBV treatment might be offered to those who persistently have an atelectasis but have no measurable effect any more. Future trials to define the role of eLVR in the treatment of COPD patients should include a surgical treatment arm in their protocol.

\section{Authors' Contributions}

F.C.T. contributed to the conception of the study, data collection, data analysis and interpretation, and drafted the manuscript. P.M.L. treated the patients, contributed to the conception of the study, data analysis and interpretation, and drafted the manuscript. D.L., F.L., C.L., M.F., R.B., and H.W. contributed to data interpretation and revised the manuscript critically for important intellectual content. P.M. and S.F. contributed to data collection, analysis and interpretation, and revised the manuscript critically for important intellectual content. All authors approved the final version of the manuscript.

Financial Disclosure and Conflicts of Interest

The authors declare that they have no conflicts of interest.

\section{References}

1 National Emphysema Treatment Trial Research Group: Patients at high risk of death after lung-volume-reduction surgery. N Engl J Med 2001; 345:1075-1083

- 2 Sciurba FC, Ernst A, Herth FJ, Strange C, Criner GJ, Marquette CH, Kovitz KL, Chiacchierini RP, Goldin J, McLennan G: A randomized study of endobronchial valves for advanced emphysema. N Engl J Med 2010; 363:1233-1244.

-3 Davey C, Zoumot Z, Jordan S, McNulty WH, Carr DH, Hind MD, Hansell DM, Rubens MB, Banya W, Polkey MI, Shah PL, Hopkinson NS: Bronchoscopic lung volume reduction with endobronchial valves for patients with heterogeneous emphysema and intact interlobar fissures (the BeLieVeR-HIFi study): a randomised controlled trial. Lancet 2015;386: 1066-1073.

4 Shrager JB: Endobronchial valves in a highly parsed emphysema population. Lancet 2015;386:1022-1023.

5 Cooper JD, Trulock EP, Triantafillou AN, Patterson GA, Pohl MS, Deloney PA, Sundaresan RS, Roper CL: Bilateral pneumectomy (volume reduction) for chronic obstructive pulmonary disease. J Thorac Cardiovasc Surg 1995;109:106-116; discussion 116-109.

-6 Herzog D, Poellinger A, Doellinger F, Schuermann D, TemmesfeldWollbrueck B, Froeling V, Schreiter NF, Neumann K, Hippenstiel S, Suttorp N, Hubner RH: Modifying post-operative medical care after EBV implant may reduce pneumothorax incidence. PLoS One 2015;10: e0128097.

-7 Schuhmann M, Raffy P, Yin Y, Gompelmann D, Oguz I, Eberhardt R, Hornberg D, Heussel CP, Wood S, Herth FJ: Computed tomography predictors of response to endobronchial valve lung reduction treatment. Comparison with Chartis. Am J Respir Crit Care Med 2015;191:767-774.

-8 Meyers BF, Yusen RD, Guthrie TJ, Patterson GA, Lefrak SS, Davis GE, Cooper JD: Results of lung volume reduction surgery in patients meeting a national emphysema treatment trial high-risk criterion. J Thorac Cardiovasc Surg 2004;127:829-835. 